\title{
Major-effect candidate genes identified in cultivated strawberry (Fragaria $\times$ ananassa Duch.) for ellagic acid deoxyhexoside and pelargonidin-3- O-malonylglucoside biosynthesis, key polyphenolic compounds
}

Jahn Davik $\mathbb{D}^{1}$, Kjersti Aaby ${ }^{2}$, Matteo Buti $\mathbb{D}^{3}$, Muath Alsheikh ${ }^{4,5}$ Nada Šurbanovski ${ }^{6}$ Stefan Martens $\mathbb{0}^{7}$, Dag Røen ${ }^{4}$ and Daniel James Sargent ${ }^{8}$

\begin{abstract}
Strawberries are rich in polyphenols which impart health benefits when metabolized by the gut microbiome, including anti-inflammatory, neuroprotective, and antiproliferative effects. In addition, polyphenolic anthocyanins contribute to the attractive color of strawberry fruits. However, the genetic basis of polyphenol biosynthesis has not been extensively studied in strawberry. In this investigation, ripe fruits from three cultivated strawberry populations were characterized for polyphenol content using HPLC-DAD-MS ${ }^{n}$ and genotyped using the iStraw35k array. GWAS and QTL analyses identified genetic loci controlling polyphenol biosynthesis. QTL were identified on four chromosomes for pelargonidin-3-O-malonylglucoside, pelargonidin-3-O-acetylglucoside, cinnamoyl glucose, and ellagic acid deoxyhexoside biosynthesis. Presence/absence of ellagic acid deoxyhexoside and pelargonidin-3-O-malonylglucoside was found to be under the control of major gene loci on LG1X2 and LG6b, respectively, on the $F$. $\times$ ananassa linkage maps. Interrogation of gene predictions in the $F$. vesca reference genome sequence identified a single candidate gene for ellagic acid deoxyhexoside biosynthesis, while seven malonyltransferase genes were identified as candidates for pelargonidin-3-O-malonylglucoside biosynthesis. Homologous malonyltransferase genes were identified in the F. $\times$ ananassa 'Camarosa' genome sequence but the candidate for ellagic acid deoxyhexoside biosynthesis was absent from the 'Camarosa' sequence. This study demonstrated that polyphenol biosynthesis in strawberry is, in some cases, under simple genetic control, supporting previous observations of the presence or absence of these compounds in strawberry fruits. It has also shed light on the mechanisms controlling polyphenol biosynthesis and enhanced the knowledge of these biosynthesis pathways in strawberry. The above findings will facilitate breeding for strawberries enriched in compounds with beneficial health effects.
\end{abstract}

Correspondence: Jahn Davik (jahn.davik@nibio.no)

'Division of Biotechnology and Plant Health, Norwegian Institute of Bioeconomy Research, Ås N-1433, Norway

${ }^{2}$ NOFIMA AS, Norwegian Institute of Food Fisheries and Aquaculture Research, Ås $\mathrm{N}-1433$, Norway

Full list of author information is available at the end of the article

\section{Introduction}

Commercial production of the cultivated strawberry (Fragaria $\times$ ananassa Duch.) has increased steadily in recent years with $\sim 12.9$ million tons of fruit sold globally in 2017 (http://www.fao.org/faostat/). Increased consumer demand for strawberries is partly due to a greater health consciousness among the consumers and an awareness of the health promoting benefits associated

\section{(c) The Author(s) 2020}

(c) (i) Open Access This article is licensed under a Creative Commons Attribution 4.0 International License, which permits use, sharing, adaptation, distribution and reproduction in any medium or format, as long as you give appropriate credit to the original author(s) and the source, provide a link to the Creative Commons license, and indicate if changes were made. The images or other third party material in this article are included in the article's Creative Commons license, unless indicated otherwise in a credit line to the material. If material is not included in the article's Creative Commons license and your intended use is not permitted by statutory regulation or exceeds the permitted use, you will need to obtain permission directly from the copyright holder. To view a copy of this license, visit http://creativecommons.org/licenses/by/4.0/. 
with the consumption of fresh fruits. Strawberries have been shown to contain a wealth of 'health promoting' compounds, many of which have been reported to play a role in reducing risk factors for cardiovascular diseases ${ }^{1,2}$. Strawberries are rich in dietary fiber, vitamin $\mathrm{C}$ and a range of secondary plant metabolites, including polyphenol compounds which exert numerous positive health benefits to consumers ${ }^{3-5}$. Ingested polyphenols are predominantly utilized in the colon, where the gut microbiota converts isoflavones, ellagitannins, and lignans to equol, urolithins, and enterolignans, respectively, which have anti-inflammatory effects and induce antiproliferative activities in humans ${ }^{4}$. Recent studies have also shown that bioactive metabolites derived from dietary polyphenols by gut microbiota exert neuroprotective effects upon crossing the blood-brain barrier ${ }^{6}$ and polyphenols have been evaluated as therapeutics for neurodegenerative diseases ${ }^{3}$.

From the perspective of breeding, polyphenolic compounds have received attention not only because of their beneficial health effects, but also because they contribute to enhanced sensorial properties of the berry experience for the consumer ${ }^{1,7,8}$. Indeed, strawberries are one of the fruits richest in ellagitannins which together with the anthocyanins and proanthocyanidins, represent the highest proportion of their polyphenol content ${ }^{9-12}$. Polyphenol compounds that accumulate in ripe strawberries include flavonoids, comprising anthocyanins, flavonols and flavan-3-ols, as well as phenolic acids and ellagitannins ${ }^{10,11}$. The anthocyanins, which are responsible for the red color of the berries, consist of four main compounds; pelargonidin-3-O-glucoside, pelargonidin-3$O$-malonylglucoside and, to a lesser extent, pelargonidin3-O-rutinoside, and cyanidin-3-O-glucoside ${ }^{10,12}$. The genetic control of anthocyanin biosynthesis has been extensively studied in the diploid strawberry species $F$. vesca. Initially, a single genetic locus $(c)$ was shown to be responsible for the yellow-fruited mutants of the species such as 'Yellow Wonder' ${ }^{13,14}$ and the gene Flavanone3-hydroylase $(F 3 H)$ in the anthocyanin biosynthesis pathway was proposed as a candidate gene for this locus ${ }^{15}$. Subsequently, however, candidate SNPs in the transcription factor FveMYB10 were confirmed to be responsible for the yellow-fruited $F$. vesca mutants ${ }^{16}$. The main flavonols present in strawberries are glycosides and glucuronides of kaempferol and quercetin. The phenolic acids are predominantly cinnamic acid derivatives, while the most abundant ellagitannin in strawberry is agrimoniin ${ }^{10}$. Although the phenolic composition of ripe strawberries has been shown to vary considerably between cultivars $^{10-12}$ and was demonstrated to be under strong genetic control in diploid Fragaria species ${ }^{17}$, knowledge of the genetic basis of polyphenol biosynthesis and accumulation in strawberry remains scarce.
In order to breed for higher concentrations of healthrelated phytochemicals such as polyphenols in the cultivated strawberry, it is first essential to understand the inheritance of such compounds. Many quantitative trait loci (QTL) mapping studies have been undertaken to investigate various aspects of cultivated strawberry fruit quality, including the identification of QTL for total anthocyanin content ${ }^{18}$, overall sweetness, titratable acidity and ascorbic acid content ${ }^{19}$, and fruit primary metabolite content $^{20}$. More recently, a study of a 'Delmarvel' $\times$ 'Selva' progeny identified many QTL similar to previous studies $^{21}$, and QTL for fruit quality have been identified in 23 related $F_{1}$ progenies using a pedigree-based approach ${ }^{22}$.

While numerous reported studies have characterized the polyphenolic content of the ripe fruit of a diverse array of strawberry cultivars ${ }^{9,11,12,23}$, the only investigation to date that has precisely characterized the ripe fruit content of individual polyphenolic compounds in a segregating mapping population was that of Urrutia et al. ${ }^{17}$ in diploid Fragaria. In that study, the authors determined the polyphenol content of the fruits of a wild diploid NIL progeny using LC-ESI-MS ${ }^{\mathrm{n}}$ and reported 76 stable QTL for the genetic control of 22 distinct polyphenolic compounds including anthocyanins, flavonols, flavan-3-ols, flavanones, hydroxycinnamic acid derivatives, and ellagitannins. However, for the most part, the QTL intervals defined in that study were large, spanning considerable physical distances on the diploid $F$. vesca genome.

The aim of this investigation was to study the inheritance of genes controlling polyphenol biosynthesis in the cultivated strawberry by characterizing the ripe fruits of three mapping populations from parental lines that had previously been shown to differ in the polyphenol content of their berries ${ }^{10}$ using HPLC-DAD-MS ${ }^{\mathrm{n}}$ against known standards. The progenies of the three mapping populations were genotyped, and GWAS and QTL analyses were performed. Following the identification of resistance QTL and genetic loci of major effect, the diploid $F$. vesca and octoploid cultivated strawberry reference genome were mined for candidate genes.

\section{Results \\ Characterization of polyphenolic compounds in parental and progeny lines}

The mean fruit concentrations for six anthocyanins, five cinnamic acids, four ellagic acid derivatives, one ellagitannin (agrimoniin), and five flavonols, are presented in Table 1. For the parents, standard errors are presented together with the marginal means to indicate the precision of the measurements, while for the $F_{1}$ hybrids, standard deviations are given to demonstrate the spread of the observations in each progeny ${ }^{24}$. A principal components plot derived from all polyphenolic compounds in the four parental cultivars (Table 1) is given in Fig. 1. 


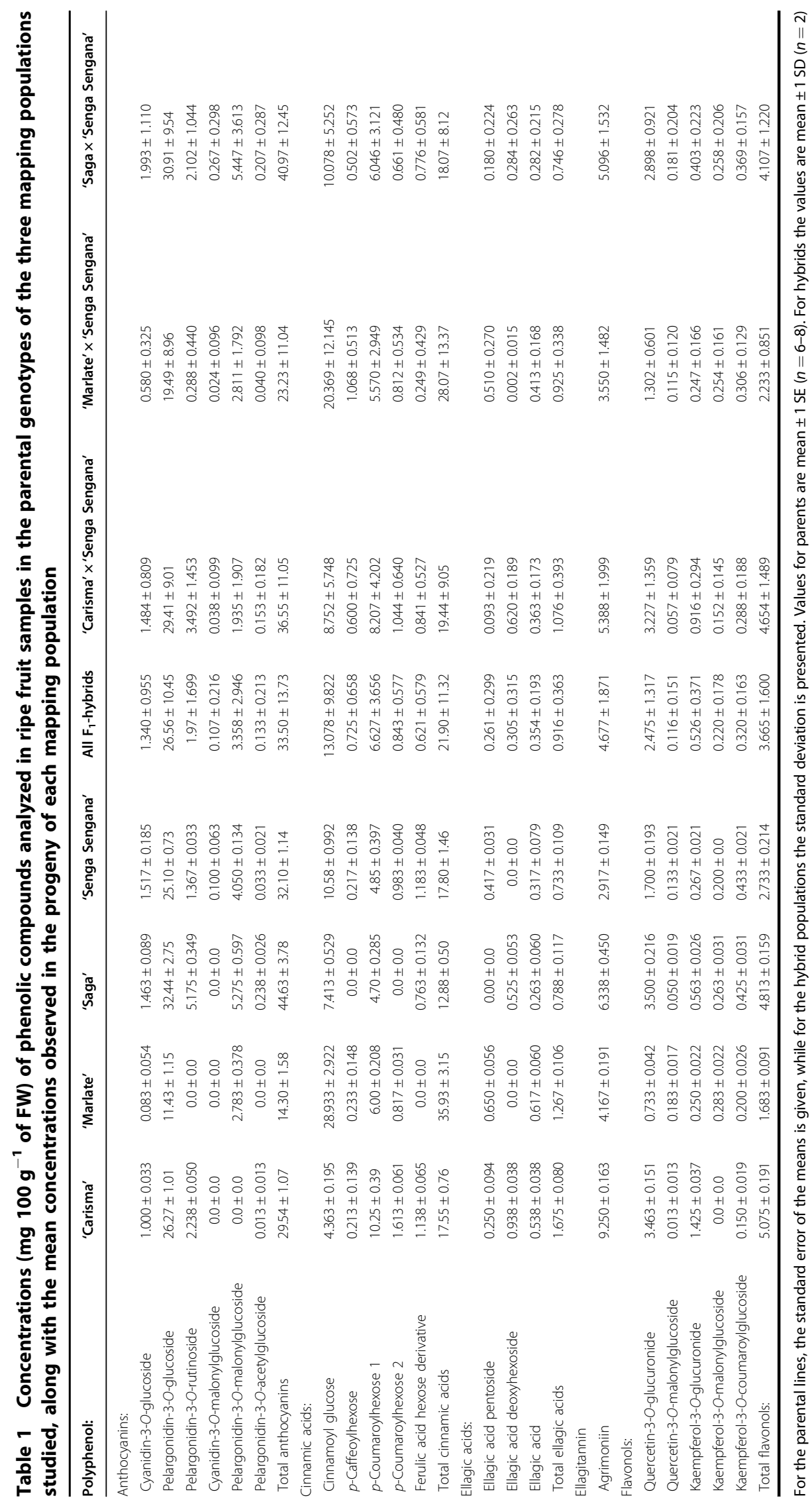




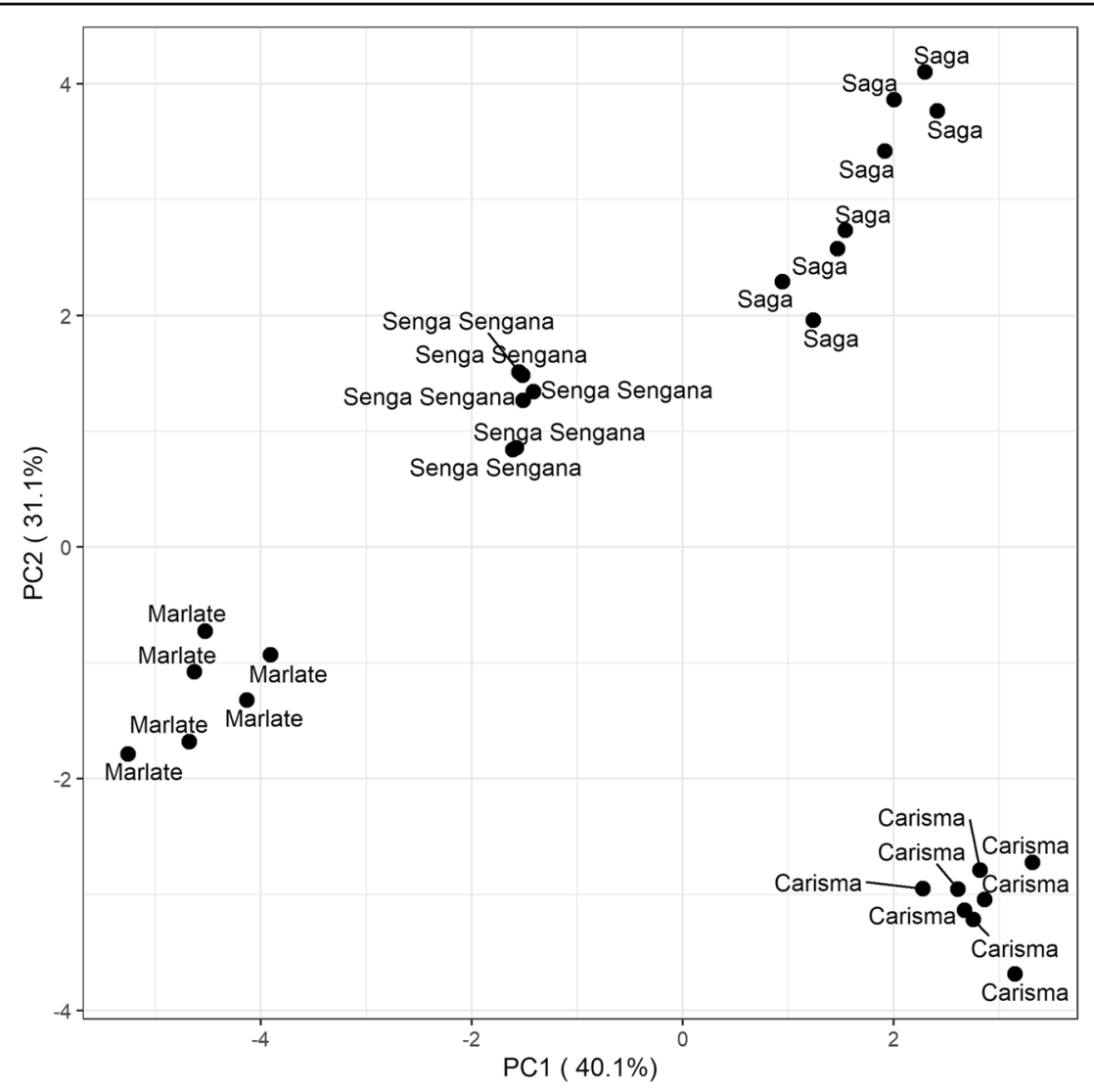

Fig. 1 Principal components plot of total polyphenol composition of the four cultivars used as parents of the mapping populations investigated. The scores of the two first principal components are plotted using standardized polyphenol concentrations of the cultivars as input

\section{Genome-wide association analysis}

GWAS were conducted using 24,062 informative markers with their relative positions derived from the $F$. vesca v4.0 reference genome ${ }^{25}$. Of these SNPs, a total of 4,317 were placed reliably on the F. $\times$ ananassa 'Camarosa' genome sequence ${ }^{26}$ by Hardigan et al. ${ }^{27}$. The GWAS analyses identified significant marker-trait associations for the polyphenols pelargonidin-3-O-malonylglucoside, pelargonidin-3-O-acetylglucoside, cinnamoyl glucose, and ellagic acid deoxyhexoside in the combined progenies dataset that exceeded the $-\log 10(p)$ value significance threshold of 6.5. A total of 163 significant associations were identified with SNP markers for pelargonidin-3-Omalonylglucoside (Fig. 2a), 60 were identified for pelargonidin-3-O-acetylglucoside (Fig. S1), 33 were identified for ellagic acid deoxyhexoside (Fig. 2b), while a single significant association was determined for cinnamoyl glucose (Fig. S1). The MLM model approach confirmed the associations observed in the basal GLM model in all four instances (Fig. S1). All significant loci identified from the 24,062 informative markers with a position on the F. vesca genome were confirmed in the 'Camarosa' reference genome (Fig. S2), however, only in the case of
pelargonidin-3-O-acetylglucoside were the most significant SNPs from the informative GWAS marker set mapped to the 'Camarosa' genome. The most significant SNPs at each locus are given in Table 2 along with the physical interval in which all significant markers were located on the $F$. vesca v4.0 reference genome.

\section{QTL analysis and mapping of traits under single major gene control}

Significant QTL were identified corresponding to the genomic intervals containing GWAS associations for pelargonidin-3-O-malonylglucoside, pelargonidin-3-Oacetylglucoside, cinnamoyl glucose, and ellagic acid deoxyhexoside (Fig. 3). A significant QTL was identified on LG1X2 of the 'Saga' $\times$ 'Senga Sengana' $(\mathrm{S} \times \mathrm{SS})$ mapping population for ellagic acid deoxyhexoside; significant QTL were identified for cinnamoyl glucose on linkage groups LG3b in the 'Carisma' $\times$ 'Senga Sengana' $(\mathrm{C} \times \mathrm{SS})$ and $\mathrm{S} \times \mathrm{SS}$ mapping populations and LG6A in the 'Marlate' $\times$ 'Senga Sengana' $(\mathrm{M} \times \mathrm{SS})$ and $\mathrm{S} \times \mathrm{SS}$ populations, and significant QTL were identified for pelargonidin-3-O-malonylglucoside and pelargonidin-3-O-acetylglucoside on LG6b of the $\mathrm{C} \times \mathrm{SS}$ and $\mathrm{S} \times \mathrm{SS}$ populations (Table 3 ). Axiom marker data 

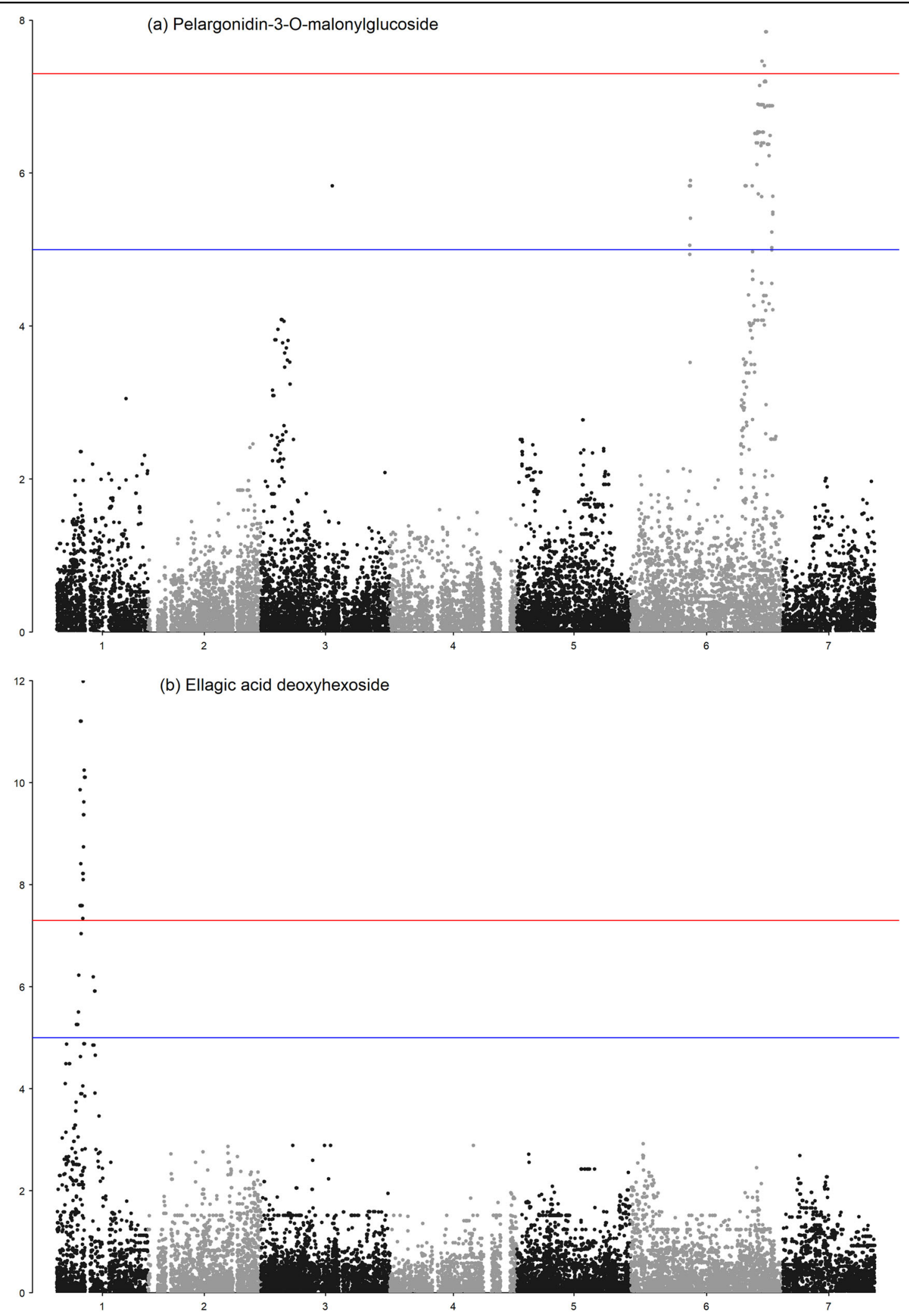

Fig. 2 Manhattan plots of markers significantly associated with pelargonidin-3-0-malonylglucoside and ellagic acid deoxyhexoside production in cultivated strawberry. A Manhattan plot constructed against the $F$. vesca v4.0 genome sequence showing marker-trait associations exceeding the $-\log 10(p)$ value significance threshold of 6.5 for the polyphenols a pelargonidin-3-O-malonylglucoside on Fvb6, and $\mathbf{b}$ ellagic acid deoxyhexoside on Fvb1 from the combined phenotypic and genotypic data from three cultivated strawberry mapping populations obtained using the MLM method 
Table 2 Position of the most significant single-nucleotide polymorphism markers from the iStraw35 array co-segregating with the polyphenols pelargonidin-3-O-malonylglucoside, pelargonidin-3-0-acetylglucoside, and ellagic acid deoxyhexoside, and the intervals with significant markers on the $F$. vesca $\mathbf{v} 4.0$ genome sequence

\begin{tabular}{|c|c|c|c|c|c|}
\hline Polyphenol & Marker & $-\log _{10}(p)$ & Chromsome & Position & Interval of significant markers according to the GWAS \\
\hline \multirow[t]{3}{*}{ Pelargonidin-3-O-malonylglucoside } & AX-166507798 & 11.22 & 6 & $34,567,280$ & $28,147,851-36,394,202$ \\
\hline & $A X-166507810$ & 11.22 & 6 & $34,693,440$ & \\
\hline & $A X-123525466$ & 11.22 & 6 & $34,624,092$ & \\
\hline \multirow[t]{2}{*}{ Pelargonidin-3-O-acetylglucoside } & $A X-166527347$ & 9.15 & 6 & $35,452,042$ & $31,813,892-36,394,202$ \\
\hline & $A X-166507856$ & 9.15 & 6 & $35,391,196$ & \\
\hline Ellagic acid deoxyhexoside & $A X-166503105$ & 11.91 & 1 & $6,707,123$ & $5,933,302-7,230,807$ \\
\hline
\end{tabular}

presented by Hardigan et al. ${ }^{27}$ enabled linkage groups on the three mapping populations to be assigned to chromosome sequences on the 'Camarosa' cultivated strawberry reference genome sequence of Edger et al. ${ }^{26}$. Between the three mapping populations, 51 markers from linkage group LG1X2 were anchored to 'Camarosa' chromosome Fvb1-3, 59 markers were anchored to chromosome Fvb3-2, 24 markers were anchored to Fvb61, and 22 markers were anchored to Fvb6-2. No ambiguities were observed within linkage groups or between homologous groups between mapping populations relating to the chromosomes to which they were anchored on the 'Camarosa' genome sequence.

Following GWAS and QTL analysis, the concentrations of ellagic acid deoxyhexoside observed in ripe fruit of the progeny of the $\mathrm{S} \times \mathrm{SS}$ population, and pelargonidin-3malonylglucoside observed in ripe fruit of the progeny of the $\mathrm{C} \times \mathrm{SS}$ and $\mathrm{S} \times \mathrm{SS}$ mapping populations were scored as a qualitative presence/absence phenotype and linkage mapping confirmed discrete genetic positions of the traits indicating that they were under the control of a single major gene in these populations. Loci controlling ellagic acid deoxyhexoside and pelargonidin-3-O-malonylglucoside biosynthesis co-segregated with markers mapped to genomic intervals on chromosome Fvb1-3 of the 'Camarosa' genome sequence between 6,921,203 bp and $7,554,779 \mathrm{bp}$ (an interval of 633,576 bp) and chromosome Fvb6-2 of the 'Camarosa' genome sequence between $31,229,150 \mathrm{bp}$ and $32,997,567 \mathrm{bp}$ (an interval of $1,768,417 \mathrm{bp}$ ), respectively (Fig. 4 ), according to the physical positions of markers with which they co-segregated. These regions corresponded to a genomic interval between $6,707,123 \mathrm{bp}$ and $8,633,460 \mathrm{bp}(1,781,033 \mathrm{bp})$ on $F v b 1$ and an interval between $34,188,443 \mathrm{bp}$ and $35,448,208$ bp $(1,259,765 \mathrm{bp})$ on $F v b 6$ of the of the $F$. vesca v4.0 genome sequence, respectively.

The QTL for pelargonidin-3-O-malonylglucoside and pelargonidin-3-O-acetylglucoside were identified in the same region of linkage group LG6b in both the $\mathrm{C} \times \mathrm{SS}$ and $\mathrm{S} \times \mathrm{SS}$ linkage maps (Fig. 3). However, when phenotypic data for pelargonidin-3-O-acetylglucoside were scored qualitatively, the segregation data did not fit the hypothesis of a single major gene controlling the biosynthesis of this compound in either population. A second significant QTL for pelargonidin-3-O-acetylglucoside biosynthesis was identified on LG6X2 in the $\mathrm{S} \times \mathrm{SS}$ mapping population. While marker genotypes were not completely predictive of phenotype, due to the genetic distance between mapped markers and the genetic loci controlling the trait variation, the combination of homozygous genotypes in this progeny of the most significantly associated markers on both LG6X2 and LG6b produced the highest and lowest concentrations of pelargonidin-3$\mathrm{O}$-acetylglucoside in the ripe fruit of the progeny (Fig. 5). The LG6X2 QTL was not recovered in the $\mathrm{C} \times \mathrm{SS}$ progeny.

Allele-effect box plots of SNP markers co-segregating in individual populations with qualitative phenotypic trait scores for ellagic acid deoxyhexoside and pelargonidin-3O-malonylglucoside (AX-166511049 and AX-166507798, respectively) are shown in Fig. 6. The allele effects for SNPs co-segregating with pelargonidin-3-O-malonylglucoside and ellagic acid deoxyhexoside were predictive of polyphenol concentrations across all three mapping populations.

\section{Candidate gene identification}

Initially, a search for candidate genes was performed within the major gene intervals identified in the $F$. vesca $\mathrm{v} 4.0$ genome sequence where a total of 561 gene predictions were identified in the $3 \mathrm{Mb}$ genomic interval (6-9 Mb) on $F v b 1$ containing the locus controlling ellagic acid deoxyhexoside biosynthesis, and 408 gene predictions were identified in the $2 \mathrm{Mb}$ genomic interval (34-36 Mb) on Fvb6 containing the locus controlling pelargonidin-3-O-malonylglucoside biosynthesis. Of those on $F v b 1$, a single gene was identified as a potential candidate for ellagic acid deoxyhexoside biosynthesis, while seven genes were identified as candidates for pelargonidin-3-O-malonylglucoside biosynthesis on $F v b 6$ (Table 4).

Gene FvH4_1g12660, located in the Fvb1 interval and annotated as a putative UDP-rhamnose: rhamnosyltransferase 1 was identified as the most likely candidate for the gene controlling ellagic acid deoxyhexoside biosynthesis. 


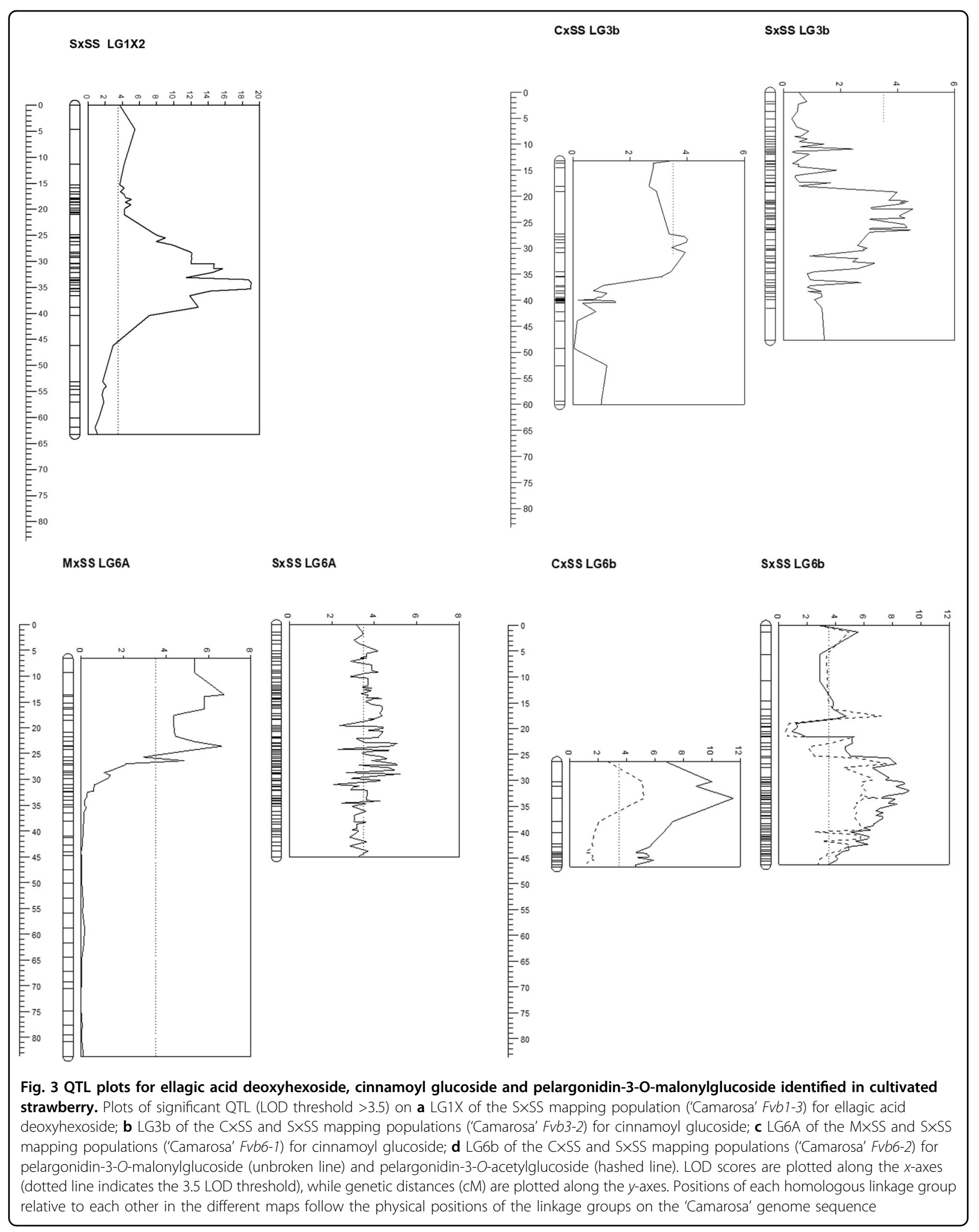




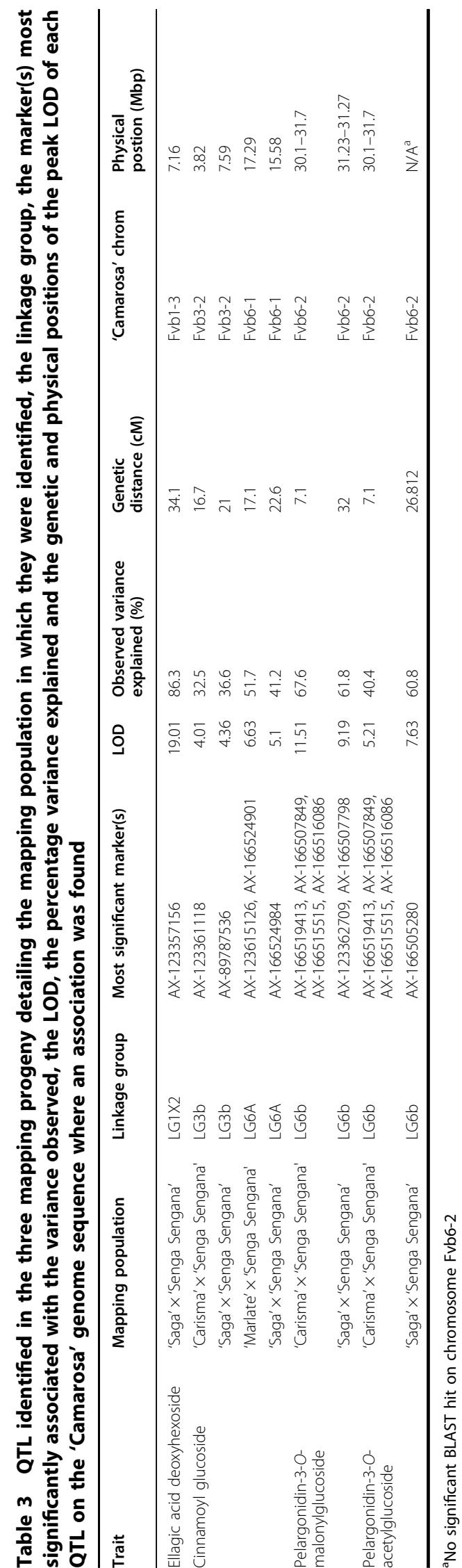

Transcript abundance data were not available for the cultivated strawberry species $F . \times$ ananassa for the candidate genes identified, however, data for the related diploid species $F$. vesca, housed on the $F$. vesca eFP browser ${ }^{16}$ (bioinformatics.towson.edu/strawberry) showed that the gene was differentially expressed during fruit development in the $F$. vesca cultivars 'Ruegen' and 'Yellow Wonder' with transcript levels observed in 'Yellow Wonder' ripe fruit higher than in 'Ruegen' (Table 5).

Within the Fvb6 interval, seven candidate genes (FvH4_6g46740.t1, FvH4_6g46741.t1, FvH4_6g46742.t1, FvH4_6g46743.t1, FvH4_6g46750.t1, FvH4_6g46770.t1, and FvH4_6g46780.t1) annotated with malonyltransferase activity were identified as likely candidates for the gene controlling pelargonidin-3-O-malonylglucoside biosynthesis. Transcript abundance data located on the strawberry eFP browser (bioinformatics.towson.edu/strawberry) ${ }^{16}$ from ripening fruit tissue of $F$. vesca cultivars 'Ruegen' and 'Yellow Wonder' was scrutinized for the seven candidate genes identified, and compared with transcript abundance data in the same tissues for gene FvH4_7g33840, the glycosyltransferase shown previously to be responsible for the production of pelargonidin-3-O-glucoside in $\mathrm{F} . \times$ ananassa and $F$. vesca ${ }^{28}$. Different transcript abundance patterns were observed for each of the candidate genes (Table 5). Genes FvH4_6g46741.t1, FvH4_6g46743.t1, FvH4_6g46750.t1, and FvH4_6g46780.t1 showed relatively high transcript levels during fruit ripening. Gene FvH4_6g46750.t1 was upregulated during ripening in both 'Ruegen' (red-fruited) and 'Yellow Wonder' (whitefruited), but more strongly upregulated in 'Ruegen', while gene FvH4_6g46780.t1 was not significantly upregulated in either cultivar, but showed significantly higher transcript levels in 'Ruegen' than in 'Yellow Wonder'. Between the same tissues, gene FvH4_7g33840 (FvGT1) was significantly upregulated during fruit development, but only in the red-fruited 'Ruegen' cultivar (Table 5).

Following identification and annotation of candidate genes in the $F$. vesca v4.0 genome sequence, gene predictions within the major gene intervals in the 'Camarosa' genome sequence were annotated, and a total of 361 gene predictions were identified in the $2.5 \mathrm{Mb}$ genomic interval (6.5-9 Mb) on Fvb1-3 containing the locus controlling ellagic acid deoxyhexoside biosynthesis, and 384 gene predictions were identified in the $2.5 \mathrm{Mb}$ genomic interval (31-33.5 Mb) on Fvb6-2 containing the locus controlling pelargonidin-3-O-malonylglucoside biosynthesis.

A search was then made for homologous genes between 'Camarosa' and $F$. vesca at the major gene loci for ellagic acid deoxyhexoside and pelargonidin-3-O-malonylglucoside biosynthesis identified using the Tripal synteny viewer implemented on the Genome Database for Rosaceae $^{29}$. The syntenic block fafvB1334 identified between chromosomes $F v b 1$ of the $F$. vesca v4.0 genome sequence 


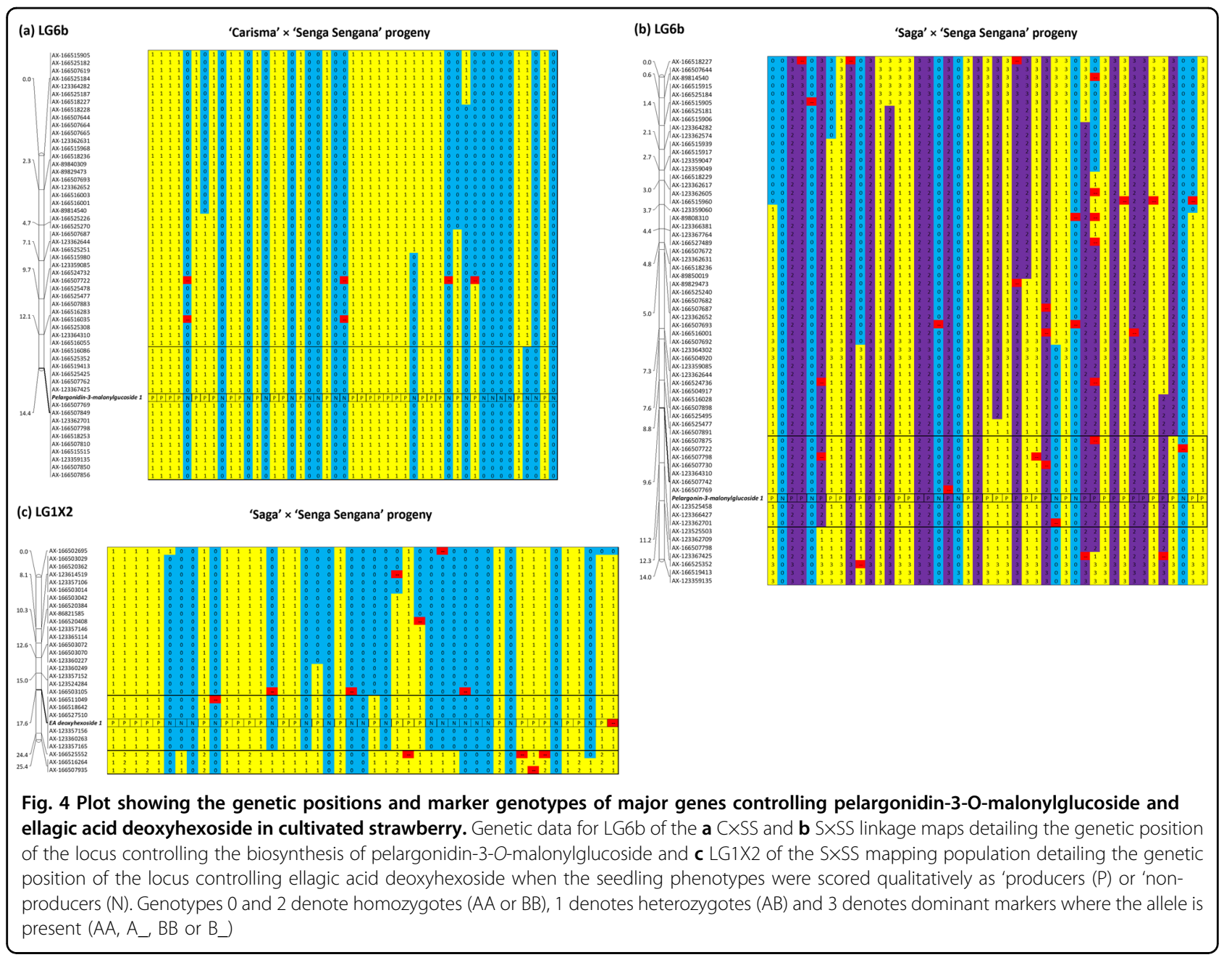

and Fvb1-3 of the 'Camarosa' genome sequence containing the gene controlling ellagic acid deoxyhexoside biosynthesis spanned the region 6,445,166-16,286,418 (10.8 Mb) in F. vesca and 5,367,671-16,828,623 (11.5 Mb) in 'Camarosa' and displayed a high degree of overall synteny (bit score $=39,011$ e-value $=0$ ). The syntenic block fafvB0858 identified between chromosomes Fvb6 of the $F$. vesca v4.0 genome sequence and $F v b 6-2$ of the 'Camarosa' genome sequence containing the gene controlling pelargonidin-3-O-malonylglucoside biosynthesis spanned the region $30,223,352-36,410,328(6.2 \mathrm{Mb})$ in $F$. vesca and 31,545,552-36,081,520 (5.5 Mb) in 'Camarosa' and also displayed a high degree of overall synteny (bit score $=24,204$ e-value $=0$ ). Within the Fvb1-3 syntenic block, there were 1076 'Camarosa' and 1378 F. vesca genes, of which 787 were identified as homologous, while in the Fvb6-2 syntenic block, there were 681 'Camarosa' and $949 \mathrm{~F}$. vesca genes, of which 492 were identified as homologous. Homologous gene sequences were identified for two of the candidate genes on Fvb6; FvH4_6g46750.1 most closely aligned to maker-Fvb6-2-snap-gene-312.67-
mRNA-1 (e-value $=1 \mathrm{e}-128)$ and FvH4_6g46740.1 most closely aligned to maker-Fvb6-2-snap-gene-312.68mRNA-1 (e-value $=0$ ). No homologous gene was identified for the candidate gene on Fvb1, FvH4_1g12660. t1_v4.0.a2.

\section{Discussion}

Polyphenol compounds are emerging as potent types of phytochemicals with pleiotropic effects on human health imparted through a dynamic interaction with the gut microbiome. Ingested polyphenols modulate microbiota community composition while microbiota enzymatically transform polyphenols into bioavailable compounds with a range of activities including anti-inflammatory and neuroprotective effects ${ }^{5}$. It is to be expected then, that fruit with increased polyphenol compounds might come into focus of breeding efforts for crops worldwide, including strawberries and other Rosaceous crops. Hence it is timely and of importance to improve our understanding of the genetics basis of polyphenol biosynthesis and accumulation in crops used for human consumption. 

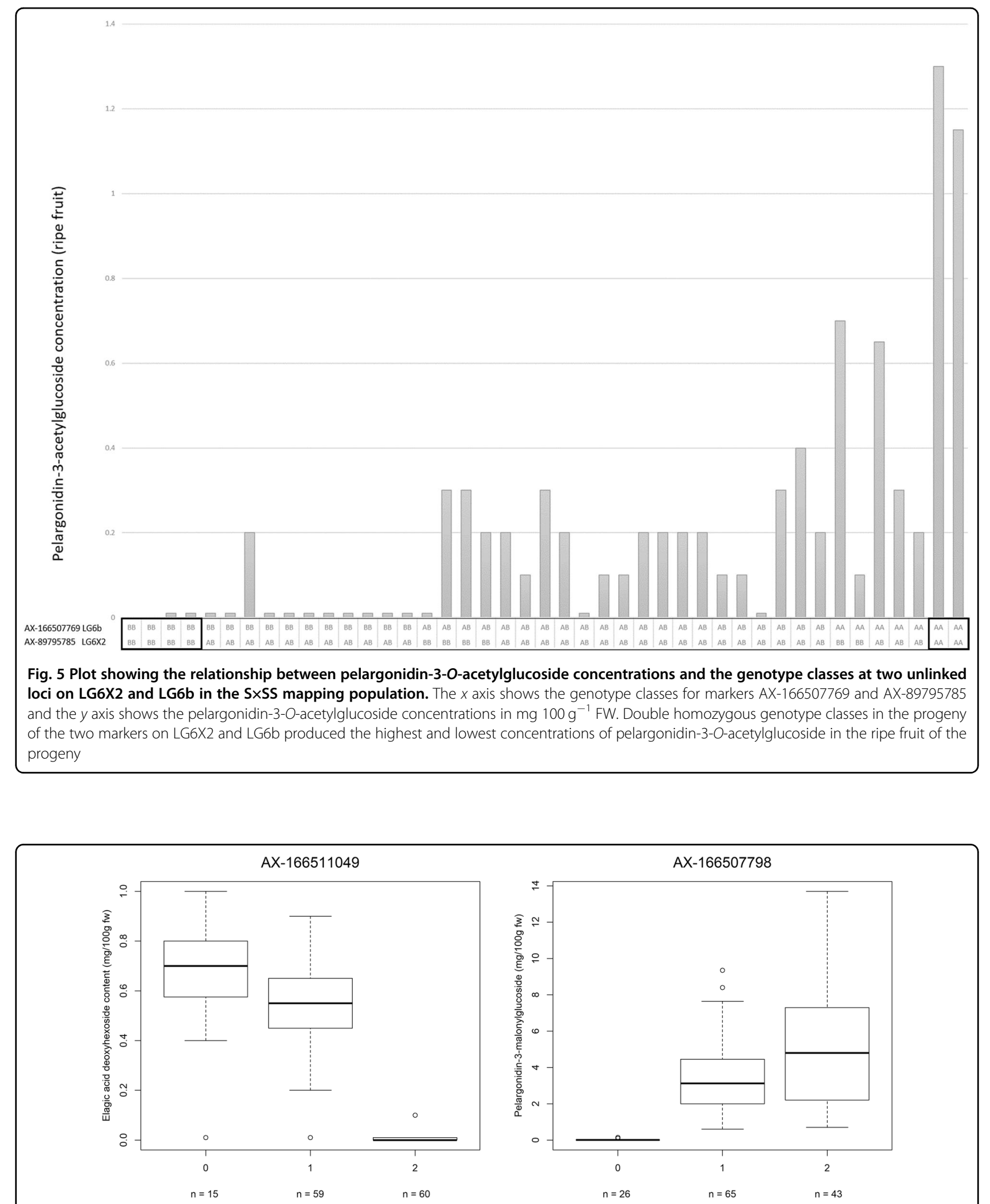

Fig. 6 Allele-effect box plots of ellagic acid deoxyhexoside and pelargonidin-3-0-malonylglucoside concentrations for two Axiom markers. SNP markers a AX-166511049 and b AX-166507798 co-segregating with quantitative phenotypic trait scores for ellagic acid deoxyhexoside and pelargonidin-3-O-malonylglucoside, respectively. Marker classes are as follows: $0=A A$ genotype, $1=A B$, and $2=B B$ genotype according to the data of Verma et al. ${ }^{51}$ 
The genetics of specific polyphenol compound biosynthesis were investigated in cultivated strawberry here for the first time using three mapping populations raised from parental genotypes previously shown to differ in the polyphenol content of their berries ${ }^{10}$. The concentrations of phenolic compounds observed in the parental lines and progenies were in the range previously reported in strawberries ${ }^{10-12}$ and significant QTL were identified for four of these polyphenols in the mapping populations studied; pelargonidin-3-O-malonylglucoside, pelargonidin-3-O-acetylglucoside, cinnamoyl glucose, and ellagic acid deoxyhexoside. Moreover, the concentrations of two of the compounds in the fruits of the mapping populations: pelargonidin-3-O-malonylglucoside and ellagic acid deoxyhexoside, were mapped as qualitative traits and were shown to be controlled by a single major gene.

\section{Genetic control of ellagic acid deoxyhexoside production}

Deoxyhexoses are a class of six-carbon monosaccharides that have had one or more of their hydroxyl groups replaced with hydrogen atoms and include rhamnose, arabinose, quinovose and fucose. Aaby et al. ${ }^{9}$ reported the presence of ellagic acid deoxyhexoside in the ripe fruits of the cultivated strawberry, with subsequent studies also reporting the presence of the compound in strawberry fruit ${ }^{10-12,30}$. Far less is known about the biosynthesis of ellagitannins than about the biosynthesis of phenylpropanoids, flavonoids, and anthocyanins. Gallic acid is the basic precursor of ellagitannin biosynthesis, and esterification of gallic acid and uridine- $5^{\prime}$-diphosphate glucose (UDP-glucose) by 1-O-acylglucose glucosyltransferases leads to the formation of $\beta$-glucogallin ${ }^{31}$, which is converted to $1,2,3,4,6$-pentagalloylglucose by 1 $O$-acylglucose dependent acyltransferases ${ }^{32}$. From here it is suggested that $3,4,5,3^{\prime}, 4^{\prime}, 5^{\prime}$-hexahydroxydiphenoyl moieties are produced through oxidation ${ }^{33}$, and that ellagic acid is then formed by hydrolysis ${ }^{34}$. A recent study in wild and cultivated Fragaria species characterized five 1-O-acylglucose glucosyltransferases ${ }^{35}$, with one gene FaGT2, physically located at $4,152,828$ bp on Fvb2 of the $F$. vesca v4.0 genome sequence, shown to be responsible for $\beta$-glucogallin biosynthesis.

To date, other genes producing proteins that catalyze reactions within the ellagitannin biosynthesis pathway have not been characterized, nor have genes responsible for the formation of ellagic acid deoxyhexoside. However, Urrutia et al. ${ }^{17}$ reported a major QTL for ellagic acid biosynthesis in diploid Fragaria in the NIL interval Fb1.26-61, which spanned the physical interval between 3,315,998 and 20,747,404 bp on the diploid Fragaria genome, and was therefore not the FaGT2 locus reported by Schulenburg et al. ${ }^{35}$. The $633 \mathrm{~kb}$ region mapped in this investigation is within the physical interval of the QTL identified by Urrutia et al. ${ }^{17}$. However, it is unlikely that 
Table 5 Relative transcript abundance of the eight candidate genes identified in this investigation in the $F$. vesca cultivars 'Ruegen' (red-fruited) and 'Yellow Wonder' (white-fruited) during fruit development

\begin{tabular}{|c|c|c|c|c|c|c|c|c|}
\hline \multirow[t]{2}{*}{ Gene } & \multicolumn{2}{|l|}{ RPKM } & \multicolumn{2}{|l|}{ RPKM } & \multicolumn{2}{|l|}{ RPKM } & \multicolumn{2}{|l|}{ RPKM } \\
\hline & Ruegen, $15 \mathrm{~d}$ & Std dev. & Ruegen, Turning & Std dev. & Yellow Wonder, 15d & Std dev. & Yellow Wonder, Turning & Std dev. \\
\hline \multicolumn{9}{|c|}{ UDP-glucose glucosyltransferase (FaGT1) } \\
\hline FvH4_7g33840 & 1.51 & 0.88 & 106.8 & 46.24 & 1.3 & 0.26 & 0.27 & 0.03 \\
\hline \multicolumn{9}{|c|}{ Pelargonidin-3-O-malonylglucoside } \\
\hline FvH4_6g46740.t1 & 0.11 & 0.05 & 0.04 & 0.01 & 0.1 & 0.03 & 0.02 & 0.02 \\
\hline FvH4_6g46741.t1 & 5.27 & 1.52 & 4.82 & 0.43 & 4.07 & 0.63 & 4.07 & 1.71 \\
\hline FvH4_6g46742.t1 & 0 & 0 & 0.02 & 0.02 & 0 & 0 & 0 & 0 \\
\hline FvH4_6g46743.t1 & 9.81 & 0.24 & 6 & 0.41 & 8.83 & 0.53 & 4.07 & 0.96 \\
\hline FvH4_6g46750.t1 & 2.98 & 0.64 & 4.29 & 0.66 & 2.32 & 0.01 & 3.17 & 1.53 \\
\hline FvH4_6g46770.t1 & 0.08 & 0.03 & 0.01 & 0.01 & 0.29 & 0.08 & 0.01 & 0.01 \\
\hline FvH4_6g46780.t1 & 5.99 & 1.46 & 6.25 & 0.17 & 3.72 & 0.47 & 3.13 & 1.88 \\
\hline \multicolumn{9}{|c|}{ Ellagic acid deoxyhexoside } \\
\hline FvH4_1g12660 & 23.05 & 8.56 & 39.61 & 8.45 & 18.39 & 0.42 & 63.34 & 2.3 \\
\hline
\end{tabular}

Data taken from the eFP browser (bioinformatics.towson.edu/strawberry) of Hawkins et al. ${ }^{10}$

the two loci are orthologous because there was no correlation between the concentrations of ellagic acid and ellagic acid deoxyhexoside in fruits of the $\mathrm{S} \times \mathrm{SS}$ mapping population. Moreover, the lack of an identifiable QTL for ellagic acid production on LG1X2 in this investigation suggests that they are under independent genetic control. Since ellagic acid and ellagic acid deoxyhexoside concentrations were not correlated, we hypothesized that the gene underlying the locus identified on LG1X2 in this investigation catalyzed the glycosylation of ellagic acid using a deoxyhexose sugar substrate, leading to the formation of ellagic acid deoxyhexoside.

Within the physical interval characterized in this investigation in $F$. vesca, a single candidate gene with a putative role in ellagic acid deoxyhexoside biosynthesis, FvH4_1g12660, was identified. Gene FvH4_1g12660 was annotated as a putative UDP-rhamnose:rhamnosyltransferase 1 , which has previously been shown to be involved in flavonoid modification in Lobelia erinus ${ }^{36}$. Rhamnose is a deoxyhexose sugar that has been shown to be present in the ripe fruits of the cultivated strawberry ${ }^{37}$. Ellagic acid rhamnosides have been identified in the stem bark of Syzygium guineese $e^{38}$ and more recently in the fruits of Rubus ulmifolius ${ }^{39}$, a close relative of the genus Fragaria. It is therefore plausible that the ellagic acid deoxyhexoside produced in cultivated strawberry is ellagic acid rhamnoside and that its biosynthesis is under the control of the candidate gene FvH4_1g12660. While caution should be exercised when comparing data between related species, the transcript abundance profile of FvH4_1g12660 in F. vesca showed that it was upregulated during fruit development, and that transcript levels in the white-fruited cultivar 'Yellow Wonder' were higher than those observed in the red-fruited cultivar 'Ruegen' (Table 5). This observation was consistent with the findings of Roy et al. ${ }^{40}$ who reported a greater accumulation of ellagitannins in white-fruited over the red-fruited $F$. vesca cultivars. Taken together, the physical location of the FvH4_1g12660 gene, and previously reported transcript profiles for $F$. vesca suggests gene FvH4_1g12660 as a candidate for ellagic acid deoxyhexoside biosynthesis in cultivated strawberry on LG1X2, particularly as no other genes in the mapping interval were potentially involved in catalyzing glycosylation reactions. However, while fulllength homologs of the FvH4_1g12660 gene were identified within the physical interval on $F v b 1$ homeologues Fvb1-1 and Fvb1-2 in the 'Camarosa' genome, the Fvb1-3 homolog of the gene was absent from the 'Camarosa' assembly. No reports have been published to date as to whether 'Camarosa' is an ellagic acid deoxyhexoside producer; if it is a non-producer, it is possible that the complete deletion of the gene from the Fvb1-3 chromosome is responsible for the lack of ellagic acid deoxyhexoside production in some cultivated strawberry accessions. Further analyses need to be performed to functionally characterize the gene and its expression in cultivated strawberry, and demonstrate if it has a role in controlling ellagic acid deoxyhexoside biosynthesis, or if another candidate gene in this region is the causal genetic agent.

\section{Genetic control of pelargonidin-3-O-malonylglucoside formation}

Anthocyanins are the class of pigments that give ripe strawberry fruits their red color and their concentrations significantly vary between cultivars. The total anthocyanin content in strawberry fruits is predominantly composed of pelargonidin-3-O-glucoside, pelargonidin3-O-malonylglucoside, cyanidin-3-O-glucoside and pelargonidin-3-O-rutinoside, and the balance between 
these anthocyanins affect the color of the ripe berries $^{23,41}$. Pelargonidin-3-O-malonylglucoside was first identified in strawberry fruits by Tamura et al. ${ }^{42}$, who noted its presence in the Japanese cultivars 'Nyoho' and 'Reiko', but did not detect it in 'Ai-berry' or 'Toyonoka'. Later, Yoshida et al. ${ }^{23}$ studied the levels of pelargonidin$3-O$-malonylglucoside in relation to fruit color in 20 cultivars of mainly Japanese origin and reported that nine of the cultivars studied were non-producers, while the remaining eleven were producers. In more recent studies, pelargonidin-3-O-malonylglucoside was determined to be the second most abundant anthocyanin in ripe red fruits of $27^{10}$ and $90^{12}$ strawberry cultivars, with concentrations ranging from 0.0 to $20.8 \mathrm{mg} 100 \mathrm{~g}^{-1}$ of FW. The results of these studies, demonstrating the presence or absence of the production of pelargonidin-3$O$-malonylglucoside between strawberry cultivars, suggests that a mutation in a single major gene is responsible for the absence of the compound in some cultivars. However, to date, the inheritance and genetic control of pelargonidin-3-O-malonylglucoside has not been studied in the cultivated strawberry.

Here, a major QTL for the presence/absence of pelargonidin-3-O-malonylglucoside was observed, and a qualitative interpretation of segregation data from two mapping populations $(\mathrm{C} \times \mathrm{SS}$ and $\mathrm{S} \times \mathrm{SS})$, demonstrated the presence of a mutation in a single major gene locus determining the lack of biosynthesis of the compound in a $1,768,417 \mathrm{bp}$ interval on $F v b 6-2$ of the $F$. vesca genome between $31,229,150$ and 32,997,567 bp. Lerceteau-Köhler et al. ${ }^{18}$ identified a QTL for total anthocyanin content on LGVIb of the 'Capitola' $\times$ 'CF1116' mapping population, and identified the microsatellite marker EMFv010 $0^{43}$ as the transferrable genetic marker most closely associated to the trait. While the precise location of the QTL was not reported in that study, the physical position of EMFv010 on Fvb6 of the $F$. vesca v4.0 genome sequence is $31,622,577 \mathrm{bp}$, which is within the genetic interval defined in this investigation. More recently, Urrutia et al. ${ }^{17}$ studied the inheritance of numerous polyphenol compounds in an interspecific diploid near isogenic line population, and mapped a minor QTL for pelargonidin-3-O-malonylglucoside explaining $10 \%$ of the observed phenotypic variation to a $6.4 \mathrm{Mb}$ genomic interval between $32,907,471$ and $39,317,498$ bp on $F v b 6$ of the $F$. vesca v4.0 genome sequence, which encompasses the interval defined in this investigation. Thus it is highly likely that an orthologous locus controls pelargonidin-3-O-malonylglucoside production in diploid and octoploid Fragaria.

Pelargonidin-3-O-malonylglucoside in cultivated strawberry is synthesized via pelargonidin, which is converted to pelagonidin-3-O-glucoside by the activity of anthocyanidin glucosyltransferase FaGT1. Subsequently, malonylation is achieved by a previously uncharacterized malonyltransferase to give pelargonidin-3-O-malonylglucoside ${ }^{44}$. Our investigation revealed that the production of pelargonidin-3-Omalonylglucoside in cultivated strawberry is the result of the action of a mutation in a single major gene which determines the qualitative presence or absence of the compound, while the concentration of pelargonidin-3-Oglucoside remains relatively unchanged. It has been previously reported that silencing of the anthocyanidin glucosyltransferase GT1 in $F . \times$ ananassa leads to a reduction in the levels of both pelargonidin-3-O-malonylglucoside and pelargonidin-3-O-glucoside in strawberry fruits, and that the transcript levels of FaGT1 increase as ripening progresses, with highest transcript abundance in ripe red berries $^{28}$. The FaGT1 locus is located on $F v b 7$ of the Fragaria genome, and is thus not the locus controlling pelargonidin-3-O-malonylglucoside biosynthesis. Given that the locus on $F v b 1$ identified in this investigation does not affect pelargonidin-3-O-glucoside biosynthesis, we postulated that the locus we identified on LG6b of the $\mathrm{S} \times \mathrm{SS}$ mapping population was likely to be the malonyltransferase gene catalyzing this final step of the pathway as described above.

The enzyme malonyl-CoA:anthocyanin 5-O-glucoside6-O-malonyltransferase was first shown to catalyze the malonylation (or aliphatic acylation) of anthocyanins in plants by Suzuki et al. ${ }^{45}$ in scarlet sage (Salvia splendens) and was more recently characterized in Arabidopsis $\left(\mathrm{At} 5 \mathrm{MAT}^{46}\right)$, where it was shown to be the gene responsible for synthesizing malonyl-modified anthocyanins. Dissection of the genetic interval on LG6b revealed a total of seven candidate genes with a putative role in polyphenol production. Each of these candidate genes was annotated as having malonyltransferase activity, with high homology to a predicted malonyl-CoA:anthocyanin 5-Oglucoside-6-O-malonyltransferase. The data of Hawkins et al. ${ }^{16}$ from previous expression analyses in cultivars of the diploid strawberry $F$. vesca showed that three of the seven candidate genes for pelargonidin-3-O-malonylglucoside biosynthesis identified here at the LG6b locus were highly expressed in ripening fruit tissues.

An evaluation of the physical region controlling pelargonidin-3-O-malonylglucoside biosynthesis on chromosome Fvb6-2 on the 'Camarosa' genome revealed two predicted genes with orthology to five of those predicted to have malonyltransferase activity from the $F$. vesca genome sequence. These three genes were the most likely candidates from the 'Camarosa' physical interval, and thus, we propose one of these three genes as the malonyltransferase that catalyzes the formation of pelargonidin-3-O-malonylglucoside from pelargonidin-3-Oglucoside. Due to the octoploid nature of the 'Camarosa' genome, further genetic characterization of this gene region, and functional characterization of these candidates in producing and non-producing $F . \times$ ananassa 
germplasm is required to demonstrate thoroughly their role in synthesizing malonyl-modified anthocyanins in the ripe fruit of cultivated strawberry.

It is also likely that one of the identified candidate genes at the Fvb6-2 locus was responsible for pelargonidin-3-Oacetylglucoside production in the $\mathrm{C} \times \mathrm{SS}$ and $\mathrm{S} \times \mathrm{SS}$ mapping populations, in tandem with a second genetic factor mapped to LG6X2 in the S×SS mapping progeny. However, since the genetic factor on LG6X2 was associated with a single Axiom marker on the $\mathrm{S} \times \mathrm{SS}$ linkage map, the genetic interval in which it resides was too large to predict likely candidate genes for its control. Further work will be required to functionally validate the role of the candidate genes on LG6b in pelargonidin-3-O-acetylglucoside production, as well as to narrow the genetic interval in which the second genetic factor is located on LG6X2 in order to identify suitable candidate genes at this locus.

\section{Materials and methods Plant material}

Four cultivars ('Carisma' ['Oso Grande' × 'Villanova'], 'Marlate' [Sel No $89258 \times 88012$ ], 'Saga' ['Korona' $\times$ ' Kimberly'], and 'Senga Sengana' ['Sieger' $\times$ 'Markee']) were chosen based on the differing content of polyphenolic compounds reported in their fruit by Aaby et al. ${ }^{10}$, and three $F_{1}$ progenies were raised using 'Senga Sengana' as the recurrent pollen parent. Three hybrid populations ('Carisma' $\times$ 'Senga Sengana' $(\mathrm{C} \times \mathrm{SS})$, 'Marlate' $\times$ 'Senga Sengana' $(\mathrm{M} \times \mathrm{SS})$, and 'Saga' $\times$ 'Senga Sengana' $(\mathrm{S} \times \mathrm{SS})$ ) were obtained through controlled crosses between parental varieties. The resultant seeds were treated with concentrated sulfuric acid for $12 \mathrm{~min}$, rinsed thoroughly in cold water before being germinated in mist chambers ( $95 \%$ relative humidity) at a photoperiod/ temperature of $16 \mathrm{~h}$ day $/ 20^{\circ} \mathrm{C}$ and $8 \mathrm{~h}$ night $/ 14{ }^{\circ} \mathrm{C}$. Artificial light was provided by high-pressure sodium lamps (SON/T, $120 \mu \mathrm{E} \mathrm{s}^{-1} \mathrm{~m}^{-2}$ ) in periods of low levels of natural light. Parental cultivars and $F_{1}$ plants from each family were propagated from runners and planted in a two-times replicated field experiment, with parental plots replicated up to four times ('Carisma' $[n=4]$, 'Marlate' $[n=3]$, 'Saga' $[n=4]$, and 'Senga Sengana' [ $n$ $=3]$ ), at the experimental site of Graminor Ltd. in Ridabu, Norway in 2015. Each plot contained six plants. Mature fruits from each plot were harvested in the peak season in 2016, flash frozen with liquid nitrogen and stored at $-80^{\circ} \mathrm{C}$ until analyzed. The number of seedlings in each mapping population were as follows: $\mathrm{C} \times \mathrm{SS}$ $(n=48) ; \mathrm{M} \times \mathrm{SS}(n=47) ; \mathrm{S} \times \mathrm{SS}(n=45)$.

\section{Chemicals used}

Quercetin-3-O-rhamnosylglucoside (rutin), gallic acid, chlorogenic acid, and ellagic acid were purchased from Sigma Aldrich Ltd. (St. Louis, MO, USA).
Pelargonidin-3-O-glucoside was purchased from Polyphenols Laboratories AS (Sandnes, Norway). Formic acid (98-100\%) and methanol were obtained from Merck KGAa (Darmstadt, Germany). Acetonitrile was sourced from VWR Chemicals (Fontenay-sous-Bois, France). All solvents were of HPLC or analytical grade, and water was of Milli-Q quality (Millipore Corp., Bedford, MA, USA).

\section{Polyphenolic compound extraction}

Fruit samples from the four parental lines and from the progeny of the three mapping populations were partially thawed and homogenized in a food processor (CombiMax 700, Braun GmbH, Kronberg, Germany). Phenolic compounds were extracted from duplicate aliquots $(10 \mathrm{~g})$ with methanol $(20 \mathrm{ml})$ by homogenization in a Polytron, PT3100 homogenizer (Kinematica AG, Littau, Switzerland) at $28,000 \mathrm{rpm}$ for $30 \mathrm{~s}$. The extracts were centrifuged at $39,200 \times g$ for $10 \mathrm{~min}$ at $20^{\circ} \mathrm{C}$ (Avanti J-26 XP Centrifuge, Beckman Coulter, USA), following which the supernatants were collected and the insoluble plant material was re-extracted as above with $70 \%$ methanol $(20 \mathrm{ml})$. The two pooled supernatants of each sample were combined, and the volume was made up to $50 \mathrm{ml}$ with $70 \%$ methanol and stored at $-80^{\circ} \mathrm{C}$ until analyzed.

\section{Polyphenolic compound analysis with HPLC-DAD-MS ${ }^{n}$}

Extracts were filtered through Millex HV $0.45-\mu \mathrm{m}$ filters (Merck Millipore Ltd., Cork, Ireland), and analyzed using an Agilent 1100 series HPLC system (Agilent Technologies, Waldbronn, Germany) equipped with an auto-sampler cooled to $4{ }^{\circ} \mathrm{C}$, a diode array detector, and a MSD XCT ion trap mass spectrometer fitted with an electrospray ionization interface as previously described ${ }^{10}$. Chromatographic separation was performed on a Synergi 4- $\mu$ m MAX RP C12 column $(250 \mathrm{~mm} \times 2.0 \mathrm{~mm}$ i.d.) equipped with a $5-\mu \mathrm{m} \mathrm{C} 12$ guard column $(4.0 \mathrm{~mm} \times 2.0 \mathrm{~mm}$ i.d.), both from Phenomenex (Torrance, CA, USA), with one mobile phase consisting of formic acid/water $(2 / 98, \mathrm{v} / \mathrm{v})$ and a second consisting of acetonitrile. Column temperature was $40^{\circ} \mathrm{C}$ and injection volume $10 \mu \mathrm{l}$. The phenolic compounds were identified based on their UV-vis spectra $(220-600 \mathrm{~nm}$ ), mass spectra and retention times relative to external standards and comparison with previous results ${ }^{10,47}$. The phenolic compounds were classified based on their characteristic UV-vis spectra and quantified by external standards. Anthocyanins were quantified against pelargonidin-3-O-glucoside (at $520 \mathrm{~nm}$ ), flavonols against rutin (at $360 \mathrm{~nm}$ ), ellagic acid and ellagic acid glycosides against ellagic acid (at $360 \mathrm{~nm}$ ), and the ellagitannin agrimoniin against gallic acid (at $260 \mathrm{~nm}$ ). Hydroxy cinnamic acids (HCA) (at $320 \mathrm{~nm}$ ) and cinnamoyl glucose (at $280 \mathrm{~nm}$ ) were quantified against chlorogenic acid (at $320 \mathrm{~nm}$ ). The results were expressed as $\mathrm{mg}$ per $100 \mathrm{~g}$ of fresh weight $(\mathrm{mg}$ $100 \mathrm{~g}^{-1}$ FW). 


\section{Phenolic compound quantification for genetic analysis}

Means and standard errors for the concentrations of all polyphenol compounds were calculated for each of the parental cultivars, while for the $\mathrm{F}_{1}$ progeny the means and standard deviations were calculated. The computations were done with the $\mathrm{R}$ function 'aggregate ${ }^{48}$. The polyphenol concentrations were scaled using the 'preProsess' function in the $\mathrm{R}$ package Caret $^{49}$ with the option 'method = scale' which divides each observation by the standard deviation of the analyzed compound. A principal component analysis (PCA) was subsequently performed on the scaled data using the prcomp package from core $\mathrm{R}$. Finally, the scores of the two first dimensions in the PCA were plotted with the ggplot package ${ }^{50}$.

\section{Molecular marker acquisition}

DNA was extracted from the parental lines and the progeny of the three mapping populations with the DNeasy Plant Minikit (Qiagen) and quality was determined using a QIAgility spectrophotometer (Qiagen). Samples passing the minimum quality threshold (a 260/280 ratio between 1.8

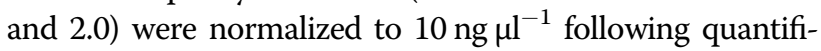
cation using a Qubit fluorometer (Thermo Scientific) against known standards. A total of 140 progenies from the three mapping populations $(\mathrm{C} \times \mathrm{SS}(n=48) ; \mathrm{M} \times \mathrm{SS}(n=$ $47) ; \mathrm{S} \times \mathrm{SS}(n=45))$ and the four parental cultivars were genotyped using the Axiom i35K strawberry array (ThermoFisher) ${ }^{51}$ on a GeneTitan instrument (ThermoFisher). Genotyping was done for each individual using the Axiom Analysis Suite software (ThermoFisher) running default quality and SNP-calling parameters.

\section{Genome-wide association analysis (GWAS)}

The segregation data were filtered with snpReady ${ }^{52}$ using a minor allele frequency of 0.05 and markers with more than $5 \%$ missing data were excluded from further analysis. Missing data were imputed using the 'knni' option in snpReady ${ }^{53}$. All data were combined into a single dataset for subsequent analysis. Since there was no a priori way of assigning all Axiom markers to the subgenomes of the F. $\times$ ananassa 'Camarosa' genome sequence $^{26}$ for GWAS analysis, the sequences of all informative iStraw35k array markers were used as queries to interrogate the $F$. vesca v4.0 genome sequence ${ }^{25}$ using a local BLAST database running default parameters. The positions determined for each marker were used for GWAS and subsequently, a subset of markers that were assigned sub-genomic locations by Hardigan et al. ${ }^{27}$ were used to create a Manhattan plot from 'Camarosa' genome sequence coordinates, and for the identification of genomic intervals for candidate gene identification. A GWAS was conducted for every polyphenol compound analyzed (Table 1) using GAPIT ${ }^{54}$, implemented in $\mathrm{R}^{48}$. The kinship matrix ${ }^{55}$ was calculated using all the useful markers, and five principal components were included as covariates. The primary model was constructed with the general linear model (GLM) algorithm ${ }^{56}$ using a minor allele frequency (MAF) of 0.05. An alternative model was developed using the mixed linear model (MLM) ${ }^{57}$ algorithm with the same covariates as with the GLM. Manhattan plots of the $-\log _{10}(p)$ values were created with the $\mathrm{R}$ package qqman ${ }^{58}$ using default settings including the 'suggestiveline' $\left(-\log _{10}(1 \mathrm{e}-5)\right)$ and 'genomewideline' $\left(-\log _{10}(5 \mathrm{e}-8)\right)$ arguments.

\section{Linkage map construction, QTL analysis, and mapping of qualitative trait loci}

Data for each mapping population were considered separately for linkage map construction. The SNP data from the parental genotypes of each progeny were scrutinized initially, and those SNPs that were heterozygous in at least one parent were retained. The remaining monomorphic SNPs, and those for which data from either parent were missing were discarded. The SNP segregation data were retained for further analysis if the progeny contained only genotypes predicted from the parental genotype combination, and for which there were $8 \%$ or fewer missing values. Linkage maps were constructed separately for each mapping progeny from all informative markers using JOINMAP 4.1 (Kyazma, NL). Marker placement was determined using regression mapping with a minimum logarithm of odds (LOD) score threshold of 3.0 , a recombination fraction threshold of 0.35 , a ripple value of 1.0, a jump threshold of 3.0 and a triplet threshold of 5.0. Mapping distances were calculated using the Kosambi mapping function, and homeologous subgenome determination (A, b, X1, and X2) for each linkage group followed the nomenclature of Sargent et al. ${ }^{59}$. The marker data of Hardigan et $\mathrm{al}^{27}$ were used to determine the 'Camarosa' chromosome homeologues for each of the identified linkage groups and subsequent BLAST analysis of specific chromosome homeologues was performed to assign a physical position to all mapped markers not placed on the 'Camarosa' genome sequence by Hardigan et al. ${ }^{27}$.

QTL analyses were performed separately for the three mapping progenies using interval mapping implemented in MAPQTL 6.0 (Kyazma, NL) with a step size of $1.0 \mathrm{cM}$, and the percentage phenotypic variance explained and associated LOD values were calculated. The linkage maps and associated LOD plots presented were plotted with MapChart 2.1 using the chart function. Following identification of significant major QTL, some traits were coded as qualitative traits and mapped as discrete genetic loci using JOINMAP 4.1 following the procedure described above. Allele-effect box plots of markers co-segregating in individual populations with qualitative phenotypic trait scores were plotted using the quantitative phenotypes for individuals in all three mapping populations and the genotypes of the most closely associated markers mapped. 


\section{Candidate gene identification and SNP characterization}

Initially, the FvH4 v4.0 a2 and 'Camarosa' gene predictions within two regions (Fvb1: 6-9 Mb and Fvb6: $34-36 \mathrm{Mb})$ of the $F$. vesca v4.0 genome sequence and (Fvb1-3: $6.5-9 \mathrm{Mb}$ and Fvb6-2: $31-33.5 \mathrm{Mb}$ ) of the 'Camarosa' genome were downloaded from the Genome Database for Rosaceae. The NCBI_nr, Araport11, Swissprot and TrEMBL annotations of all genes within both intervals were scrutinized (https://www.rosaceae.org/analysis/252) ${ }^{29}$, following which the predicted coding sequences (CDS) were imported into OmicsBox (https://www.biobam.com/ omicsbox), and additional information (GO annotations, Interpro information, EggNOG GOs) was added, KEGG pathway information was obtained and candidate genes were identified. Subsequently, synteny comparison between the F. vesca and 'Camarosa' genomes was performed using the Tripal synteny viewer implemented on the Genome Database for Rosaceae ${ }^{29}$ producing synteny comparisons for all genes within the homologous regions identified. Gene transcript data in F. vesca fruits during ripening for the candidate genes identified was retrieved from the strawberry (F. vesca) eFP Browser (http://mb3.towson.edu/efp/ cgi-bin/efpWeb.cgi) ${ }^{16}$.

\section{Acknowledgements}

J.D., K.Aa., M.A., and D.R. were supported by grant \#234312/E50 to the project 'High quality cultivars of strawberry and raspberry for processing and fresh market' from the Norwegian Research Council.

\section{Author details}

'Division of Biotechnology and Plant Health, Norwegian Institute of Bioeconomy Research, Ås N-1433, Norway. ${ }^{2}$ NOFIMA AS, Norwegian Institute of Food Fisheries and Aquaculture Research, Ås N-1433, Norway. ${ }^{3}$ Department of Agriculture, Food, Environment and Forestry, University of Florence, Florence, Italy. ${ }^{4}$ Graminor Breeding Ltd., N-2322 Ridabu, Norway. ${ }^{5}$ Department of Plant Sciences, Norwegian University of Life Sciences, Ridabu N-1432 Ås, Norway. ${ }^{6}$ NIAB-EMR, East Malling, ME19 6BJ Kent, UK. ${ }^{7}$ Department of Food Quality and Nutrition, Fondazione Edmund Mach, Centro Ricerca e Innovazione, Via E. Mach 1, 38010 San Michele all'Adige, TN, Italy. ${ }^{8}$ Department of Genetics, Genomics and Breeding, NIAB-EMR, East Malling, ME19 6BJ Kent, UK

\section{Author contributions}

J.D. conceived the study, performed experimentation, analyzed data, and authored the paper; K.A. performed experimentation, analyzed data, and critically reviewed the paper; M.B. analyzed data and co-authored the paper; M.A. conceived the study and critically reviewed the paper; N.S. analyzed data and co-authored the paper; S.M. critically evaluated the data analysis and results and co-authored the paper; D.R. conceived the study and critically reviewed the paper; D.J.S. performed experimentation, analyzed the data, and authored the paper. All authors read and approved the final paper.

\section{Data availability}

If the paper is accepted, data will be made available as supplementaries.

\section{Conflict of interest}

The authors declare that they have no conflict of interest.

Supplementary Information accompanies this paper at (https://doi.org/ 10.1038/s41438-020-00347-4).

Received: 7 April 2020 Revised: 27 May 2020 Accepted: 31 May 2020 Published online: 01 August 2020

\section{References}

1. Giampieri, F. et al. Strawberry as a health promoter: an evidence based review. Food Funct. 6, 1386-1398 (2015)

2. Basu, A. et al. Freeze-dried strawberries lower serum cholesterol and lipid peroxidation in adults with abdominal adiposity and elevated serum lipids. J. Nutr. 144, 830-837 (2014).

3. Renaud, J. \& Martinoli, M.-G. Considerations for the use of polyphenols as therapies in neurodegenerative diseases. Int. J. Mol. 20, 1883 (2019).

4. Ercolini, D. \& Fogliano, V. Food design to feed the human gut microbiota. J. Agric. Food Chem. 66, 3754-3758 (2018).

5. Filosa, S., Di Meo, F. \& Crispi, S. Polyphenols-gut microbiota interplay and brain neuromodulation. Neural Regen. Res. 13, 2055-2059 (2018).

6. Figueira, I. et al. Polyphenols journey through blood-brain barrier towards neuronal protection. Sci. Rep. 7, 11456 (2017).

7. Edirisinghe, I. \& Burton-Freeman, B. Anti-diabetic actions of berry polyphenols - Review on proposed mechanisms of action. J. Berry Res. 6, 237-250 (2016).

8. Li, D., Wang, P., Luo, Y., Zhao, M. \& Chen, F. Health benefits of anthocyanins and molecular mechanisms: Update from recent decade. Crit. Rev. Food Sci. Nutr. 57, 1729-1741 (2017).

9. Aaby, K., Ekeberg, D. \& Skrede, G. Characterization of phenolic compounds in strawberry (Fragaria $\times$ ananassa) fruits by different HPLC detectors and contribution of individual compounds to total antioxidant capacity. J. Agric Food Chem. 55, 4395-4406 (2007).

10. Aaby, K., Mazur, S., Nes, A. \& Skrede, G. Phenolic compounds in strawberry (Fragaria $\mathrm{x}$ ananassa Duch.) fruits: composition in 27 cultivars and changes during ripening. Food Chem. 132, 86-97 (2012).

11. Buendía, B. et al. HPLC-MS analysis of proanthocyanidin oligomers and other phenolics in 15 strawberry cultivars. J. Agric. Food Chem. 58, 3916-3926 (2010)

12. Nowicka, A., Kucharska, A. Z., SokółŁętowska, A. \& Fecka, I. Comparison of polyphenol content and antioxidant capacity of strawberry fruit from 90 cultivars of Fragariaxananassa Duch. Food Chem. 270, 32-46 (2019).

13. Brown, T. \& Wareing, P. F. The genetic control of the everbearing habit and three other characters in varieties of Fragaria vesca. Euphytica 14,97-112 (1965).

14. Davis, T. M. \& Yu, H. A linkage map of the diploid strawberry, Fragaria vesca. J. Hered. 88, 215-221 (1997)

15. Deng, C. \& Davies, T. M. Molecular identification of the yellow fruit color (c) locus in diploid strawberry: a candidate gene approach. Theor. Appl. Genet. 103, 316-322 (2001).

16. Hawkins, C. et al. An eFP browser for visualizing strawbern fruit and flower transcriptomes. Hort. Res. 4, 17029 (2017).

17. Urrutia, M., Schwab, W., Hoffmann, T. \& Monfort, A. Genetic dissection of the (poly)phenol profile of diploid strawberry (Fragaria vesca) fruits using a NIL collection. Plant Sci. 242, 151-168 (2016).

18. Lerceteau-Kohler, E. et al. Genetic dissection of fruit quality traits in the octoploid cultivated strawberry highlights the role homoeo-QTL in their control. Theor. Appl. Genet. 124, 1059 (2012)

19. Zorilla-Fontanesi, Y. et al. Development and bin mapping of strawberry genicSSRs in diploid Fragaria and their transferability across the Rosoideae subfamiliy. Mol. Breed. 27, 137-156 (2011).

20. Vallarino, J. G. et al. Identification of quantitative loci and candidate genes for primary metabolite content in strawberry fruit. Hort. Res. 6, 4 (2019).

21. Castro, P. \& Lewers, K. S. Identification of quantitative trait loci (QTL) for fruitquality traits and number of weeks of flowering in the cultivated strawberry. Mol. Breed. 36, 138 (2016).

22. Verma, S. et al. Clarifying sub-genomic positions of QTLs for flowering habit and fruit quality in U.S. strawberry (Fragaria $\times$ ananassa) breeding populations using pedigree-based QTL analysis. Hort. Res. 4, 17062 (2017).

23. Yoshida, Y., Goto, T., Hirai, M. \& Masuda, M. Anthocyanin accumulation in strawberny fruits as affected by nitrogen nutrition. Acta Hort. 567, 357-360 (2002).

24. Altman, D. G. \& Bland, J. M. Standard deviations and standard errors. BMJ 331, 903 (2005)

25. Edger, P. P. et al. Single-molecule sequencing and optical mapping yields an improved genome of woodland strawberry (Fragaria vesca) with chromosome-scale contiguity. Gigascience 7, 1-7 (2018).

26. Edger, P. P. et al. Origin and evolution of the octoploid strawberry genome Nat. Genet. 51, 541-547 (2019). 
27. Hardigan, M. A. et al. Genome synteny has been conserved among the octoploid progenitors of cultivated strawberry over millions of years of evolution. Front. Plant Sci. 10, 1789 (2020).

28. Griesser, M. et al. Redirection of flavonoid biosynthesis through the downregulation of an anthocyanidin glucosyltransferase in ripening strawberry fruit. Plant Physiol. 146, 1528-1539 (2008).

29. Jung, $\mathrm{S}$. et al. 15 years of GDR: new data and functionality in the Genome Database for Rosaceae. Nucleic Acids Res. 47, D1137-D1145 (2019).

30. Kosinska, A., Diering, S., Prim, D., Héritier, J. \& Andlauer, W. Phenolic compounds profile of strawberry fruits of Charlotte cultivar. J. Berry Res. 3, 15-23 (2013).

31. Niemetz, R. \& Gross, G. G. Gallotannin biosynthesis: $\beta$-glucogallin: hexagalloyl 3-O-galloyltransferase from Rhus typhina leaves. Phytochemistry 58, 657-661 (2001).

32. Hagenah, S. \& Gross, G. G. Biosynthesis of 1,2,3,6-tetra-O-galloyl- $\beta$-d-glucose Phytochemistry 32, 637-641 (1993).

33. Niemetz, R. \& Gross, G. G. Enzymology of gallotannin and ellagitannin biosynthesis. Phytochemistry 66, 2001-2011 (2005).

34. Niehaus, J. U. \& Gross, G. G. A gallotannin degrading esterase from leaves of pedunculate oak. Phytochemistry 45, 1555-1560 (1997).

35. Schulenburg, K. et al. Formation of $\beta$-glucogallin, the precursor of ellagic acid in strawberry and raspberry. J. Exp. Bot. 67, 2299-2308 (2016).

36. Hsu, Y.-H. et al. Functional characterization of UDP-rhamnose-dependent rhamnosyltransferase involved in anthocyanin modification, a key enzyme determining blue coloration in Lobelia erinus. Plant J. 89, 325-337 (2017).

37. Akšić, F. M. et al. Comparison of sugar profile between leaves and fruits of blueberry and strawberry cultivars grown in organic and integrated production system. Plants 8, 205 (2019).

38. Desire Djoukeng, J., Abou-Mansour, E., Azefek Tapondjou, L., Lontsi, D. \& Tabacchi, R. Identification of ellagic acid derivatives from stem bark of Syzygium guineense (Myrtaceae). Nat. Prod. Comm. 2, 261-263 (2007).

39. Fontaine, B. M. et al. Identification of ellagic acid rhamnoside as a bioactive component of a complex botanical extract with anti-biofilm activity. Front. Microbiol. 8, 1-11 (2017)

40. Roy, S., Wu, B., Liu, W. \& Archbold, D. D. Comparative analyses of polyphenolic composition of Fragaria spp. color mutants. Plant Physiol. Biochem. 125, 255-261 (2018)

41. Muñoz, C. et al. Polyphenol composition in the ripe fruits of Fragaria species and transcriptional analyses of key genes in the pathway. J. Agric. Food Chem. 59, 12598-12604 (2011)

42. Tamura, H., Takada, M. \& Yoshida, Y. Pelargonidin 3-O-(6-O-Malonyl-B-D-glucoside) in Fragaria $x$ ananassa Duch. cv. Nyoho. Biosci. Biotech., Biochem. 59 1157-1158 (1995).

43. James, C. M., Wilson, F., Hadonou, A. M. \& Tobutt, K. R. Isolation and characterization of polymorphic microsatellites in diploid strawberry (Fragaria vesca L.) for mapping, diversity studies and clone identification. Mol. Ecol. Notes 3, 171-173 (2003)

44. Härtl, K. Formation of Ellagic Acid Precursors in Fragaria. PhD Thesis, Technische Universität München, 118 (2017)

45. Suzuki, $H$. et al. Malonyl-CoA:anthocyanin 5-O-glucoside-6"'-O-malonyltransferase from Scarlet Sage (Salvia splendens) flowers: ENZYME PURIFICATION, GENE CLONING, EXPRESSION, AND CHARACTERIZATION. J. Biol. Chem. 276, 49013-49019 (2001).

46. D'Auria, J. C., Reichelt, M., Luck, K., Svatoš, A. \& Gershenzon, J. Identification and characterization of the BAHD acyltransferase malonyl COA: anthocyanidin 5-Oglucoside-6"-O-malonyltransferase (At5MAT) in Arabidopsis thaliana. FEBS Lett. 581, 872-878 (2007)

47. Aaby, K., Skrede, G. \& Wrolstad, R. E. Phenolic composition and antioxidant activities in flesh and achenes of strawberries (Fragaria ananassa). J. Agric. Food Chem. 53, 4032-4040 (2005).

48. R Core Team. R: A Language and Programming Environment for Statistical Computing (2016).

49. Kuhn, M. Caret: Classification and Regression Training. R package version 6.0-85. https://CRAN.R.project.org/package= caret (2020).

50. Wickham, H. ggplot2: Elegant Graphics for Data Analysis (Springer-Verlag, New York, 2016).

51. Verma, S. et al. Development and evaluation of the Axiom IStraw35 384HT array for the allo-octoploid cultivated strawberry Fragaria $\times$ ananassa. Acta Hort. 1156, 75-82 (2017).

52. Granato, I. \& Fritsche-Neto, R. snpReady: preparing genotypic datasets in order to run genomic analysis. R package version 0.9.6. https://CRAN.R-project.org/ package $=$ snpReady $(2018)$

53. Hastie, T., Tibshirani, R., Narasimhan, B. \& Chu, G. impute: Imputation for Microarray Data. R package version 1.60 .0 (2019).

54. Tang, $Y$. et al. GAPIT Version 2: an enhanced integral tools for genomic association and prediction. Plant Genome 9, 1-9 (2016).

55. VanRaden, P. Efficient methods to compute genomic predictions. J. Dairy Sci. 91, 4414-4423 (2008)

56. Lipka, A. E. et al. GAPIT: genome association and prediction integrated tool. Bioinformatics 28, 2397-2399 (2012)

57. $Y u, J . M$. et al. A unified mixed-model method for association mapping that accounts for multiple levels of relatedness. Nat. Genet. 38 203-208 (2006).

58. Turner, S. D. qqman: Q-Q and Manhattan plots for GWAS data. R package version 0.1.4. biorXiv https://CRAN.R-project.org/pacakge=qqman (2017).

59. Sargent, D. J. et al. HaploSNP affinities and linkage map positions illuminate subgenome composition in the octoploid, cultivated strawberry (Fragaria $\mathrm{x}$ ananassa). Plant Sci. 242, 140-150 (2016). 